\title{
Modelo de Gestión para proyectos interdisciplinarios de Vinculación
}

\section{Management model for interdisciplinary community outreach projects}

\section{JOSÉ ERNESTO PAZMIÑO ENRÍQUEZ*, LUIS ENRIQUE CORTEZ ALVARADO, LINDA CELESTE MALDONADO GUERRERO}

Universidad Laica Vicente Rocafuerte de Guayaquil, Av. De las Américas No. 70 frente al Cuartel Modelo, Guayaquil, Ecuador *ipazminoe@ulvr.edu.ec

\section{RESUMEN}

La Universidad Laica Vicente Rocafuerte de Guayaquil (ULVR) y su Departamento de Vinculación con la Sociedad (DVS), en busca de la mejora continua en el desarrollo de sus procesos académicos diseña un Modelo de Gestión para la vinculación con la sociedad, denominado DVS-ULVR, el mismo que recoge las aristas necesarias para alcanzar estándares de calidad de la extensión universitaria, alineados a los objetivos instituciones y la atención a las necesidades de la sociedad. Las zonas de intervención de la universidad de acuerdo con la ley son la 5 y 8 , y sus dominios se encuentran centrados en sus Facultades de Administración, Educación, Ingeniería, Industria y Construcción, y Ciencias Sociales y Derecho. Para alcanzar el éxito en las labores comunitarias que desarrolla la universidad, de acuerdo con lo establecido por los organismos que controlan la educación superior, fue necesaria la construcción de un Modelo de Gestión para la vinculación que se articule a los requerimientos de la sociedad y de la institución. Se analizaron las bases teóricas elaboradas por actores académicos de la gestión social, que han desarrollado sus propios modelos lo que permite resolver problemas en distintos países, y por sus características sirvieron de guía para la construcción del modelo.

Este se estructura sobre ocho pilares fundamentales, siendo estos: la unificación de criterios, la planeación de los proyectos de vinculación, la elaboración de formatos, la medición de los resultados, la relación de estos con las líneas de investigación y vinculación universitaria, la dirección de la gestión, la implantación de los sistemas de control y los procesos de retroalimentación. Durante el año 2018, la ULVR, realizó la aplicación del modelo desarrollado y se presentan en este artículo, los resultados obtenidos de forma general.

Palabras claves

Evaluación, herramientas metodológicas, indicadores, interdisciplinario, Modelo de gestión 


\section{ABSTRACT}

Laica Vicente Rocafuerte of Guayaquil University and the Community Engagement Department, in constant search of improving their academic processes has designed the Community Engagement Management Model, under the name of "DVS-ULVR". This document compiles every necessary aspect in order to achieve high quality standards in University Extension and it is aligned with institutional objectives and permanent attention to the community necessities. According to the LOES (Superior Education Organic Law), the areas of influence for ULVR are numbers 5 and 8, and its area of expertise is concentrated on its four Faculties: Administration, Education, Engineering and Construction, and Social Sciences and Law. In order to succeed in Community Engagement activities, according to what is established by the entities that supervise and control Superior Education, it was necessary the creation of this Management Model lined up at the same time with the institutional and society requirements. Various experts in the topic have created their own models focused on solving specific problems in their countries. These theories were analyzed and due to their characteristics served as a guide in the assembly of the Model. Community Engagement Management Model is supported on eight fundamental pillars: Unified criteria, Community Engagement Project planning, Model formats design, Outcome measure, Relation with research lines, Process management, Control system and feedback. In 2018, ULVR implemented this new created model and their results are presented in this article.

Key words

Evaluation, Indicators, Interdisciplinary, Management Model, Methodological tools

\section{INTRODUCCIÓN}

Las universidades ecuatorianas asumen la ejecución de diversos proyectos de vinculación con la colectividad como parte de su responsabilidad social, con el objetivo de contribuir a la transformación de la sociedad. La Universidad Laica Vicente Rocafuerte de Guayaquil, desarrolla acciones puntuales con tal fin, entre ellas se destaca la propuesta de proyectos interdisciplinarios con la participación de las diferentes Unidades Académicas, lo que presupone un salto cualitativamente superior al transitar de proyectos específicos de cada una de las carreras a propuestas de mayor integralidad; ello demanda del análisis de procesos realizados en periodos anteriores para su mejoramiento constante, además de establecer las vías para la concepción de estos, en correspondencia con los nuevos objetivos institucionales, cuya legalidad está respaldada por el cumplimiento de las líneas de investigación de la ULVR, las cuales direccionan las líneas de vinculación.

La realización de este trabajo requirió un análisis de la forma que adoptaba cada uno de los Departamentos para la elaboración de sus propios lineamientos, ajustados a propuestas viabilizadas por organismos de control en otras etapas. La relación entre Docencia, Investigación y Vinculación con la Sociedad demanda de la actualización de las líneas de investigación y vinculación, las cuales poseen un carácter orientador para el desarrollo de diferentes proyectos en el contexto universitario con independencia de su naturaleza.

Este planteamiento sirvió para que el Departamento de Vinculación, cumpliendo los ejes establecidos en la LOES, adopte las líneas de 
investigación de la Universidad para los proyectos de vinculación, lo que conllevó a la toma de decisiones favorecedoras de la aplicación de proyectos interdisciplinarios, por cada una de las Facultades de la Universidad.

Esta decisión condujo a la propuesta de un mecanismo funcional para todos los actores de la vinculación, así como sus beneficiarios, en aras del establecimiento de compromisos en la ejecución y obtención de los resultados.

La aplicación del procedimiento de análisis de contenido, de la obra de diferentes autores, resultó de gran importancia en la valoración de diversos sistemas de gestión social, con el objetivo de conocer su estructuración y funcionamiento, así como las vías utilizadas para dar solución a problemas de este tipo.

La sistematización realizada y el resultado de diferentes talleres permitieron el diseño de un Modelo de Gestión de vinculación para la ULVR, este modelo recoge las aportaciones de estos autores y se construye con los sustentos necesarios para su aplicación. Para ello, durante el año 2018 se elaboraron las guías, instrumentos y las herramientas metodológicas necesarias para su implementación, las cuales se aplican en los proyectos durante este mismo año.

El seguimiento y control de los proyectos elaborados permitió una validación preliminar del modelo propuesto. Los resultados obtenidos en cada uno de los proyectos revelan como el modelo construido permite a nuevos actores sociales contar con una propuesta para aplicar sus proyectos y llevarlos hacia la obtención de sus objetivos propuestos.

\section{MARCO TEÓRICO}

Para sustentar teóricamente esta investigación, referente al Modelo de Gestión de vinculación universitaria, hemos partido de la realidad generada a través de los proyectos realizados por las Carreras que oferta la Universidad Laica Vicente Rocafuerte de Guayaquil. En este proceso se tuvo la necesidad de implantar un modelo de gestión, el mismo que se construyó sobre la base de modelos de gestión social, que permitieron viabilizar la construcción de nuestro modelo, ajustado a la realidad de la educación superior ecuatoriana.

Tres fueron los modelos utilizados para esta construcción académica: el primero es uno desarrollado en Venezuela por el Instituto Universitario de Tecnología José Antonio Anzoátegui, donde (Royero, 2009) lo define como un conjunto de procesos que parten de las principales áreas claves dentro del sistema organizacional, con el fin de diseñar indicadores y estándares basados en los planes y programas estratégicos intentados por la organización; el segundo Modelo, desarrollado por Fantova (Fantova, 2005) se fundamenta la gestión en tres ejes: la planificación, la interlocución y la evaluación; y el tercero es el Modelo de planeación y control integral de la gestión municipal desarrollado por (Martínez Fajardo, 2004) en Colombia sobre la eficacia que se puede lograr en programas de acción social con su aplicación.

\section{Modelo Integral de Control de Gestión (MICG)}

(Royero, 2009) señala que este modelo permite establecer indicadores cuantitativos y cualitativos que deben ser medidos por índices de desempeño y cuadros de mando, que permitan 
monitorear efectivamente su cumplimiento, el cual se estructura de la siguiente manera: Planeación del sistema a controlar; Identificación de áreas claves, variables y procesos críticos; Diseño del sistema de indicadores; Diseño del o los instrumentos de control; Diseño de la presentación de la información; e, Implantación del sistema de control.

De acuerdo con (Royero, 2009) en cada una de las etapas se deben diseñar manuales de procedimientos o instructivos que permitan aplicar cada una de las fases. Para la de diagnóstico, es vital contar con un manual de aplicación, organización, tabulación, análisis y presentación de los resultados. En la fase de identificación de las áreas críticas, debe elaborarse un manual, donde se destaque la metodología básica para identificar las áreas y factores críticos del éxito. En la fase de indicadores, será necesario estructurar un manual sobre la base del tipo de indicador, su función dentro del sistema, sus unidades de medida, la forma de cálculo, la frecuencia de su medición, un manual de la información, y los rangos, así como los usuarios de este.

Para la fase de implantación, un manual donde se indiquen la estructura organizativa necesaria, los perfiles de los sujetos responsables de operar el sistema y los procedimientos para la administración del sistema de control (Royero, 2009).

\section{Modelo Fantova}

Fernando Fantova (Fantova, 2005) propone un modelo para la gestión de la intervención social, señalando que sus procesos básicos son fundamentales y primarios, que aparecen ante un hipotético desarrollo de la gestión a partir de los desarrollos operativos. Se trata de tres instancias generadas de forma permanente y simultánea en la organización, obedeciendo a un patrón cíclico: planificacióninterlocución-evaluación y así sucesivamente.

Estos tres procesos son asumidos en su responsabilidad por parte de una sola persona, quien gobierna el equipo central y distribuye las funciones y directrices a seguir para el correcto desenvolvimiento de la gestión (figura 1).

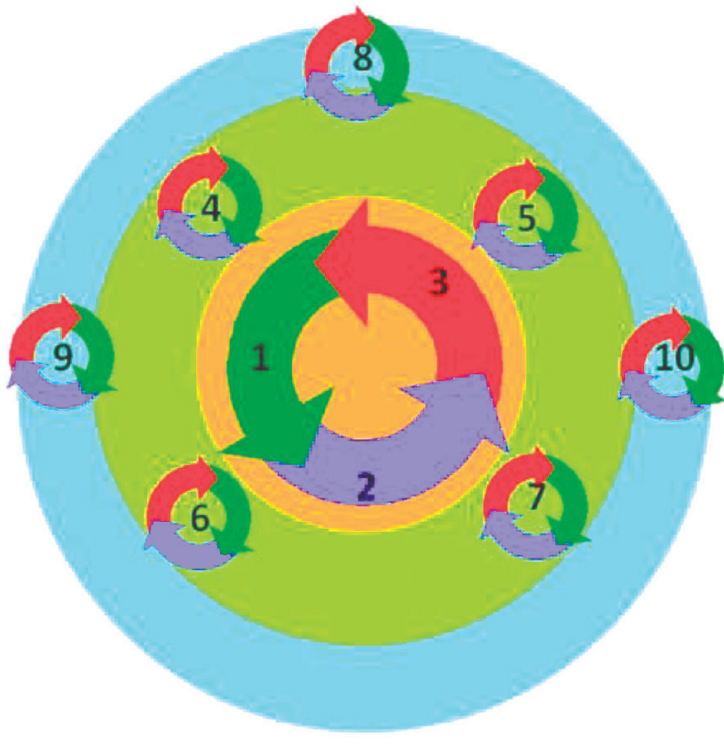

1. Planificación

2. Interlocución

3. Evaluación

4. Gestión de recursos humanos

5. Gestión económicofinanciera

6. Gestión de la información

7. Gestión de recursos materiales

8. Gestión de las relaciones

9. Gestión de la estructuración

10. Gestión de aprendizaje
Figura 1. Procesos básicos de organización. Fuente: (Fantova, 2005) 
Los tres procesos básicos son: planificación (1), interlocución (2) y evaluación (3). Adicionalmente, este modelo tiene cuatro procesos vinculados a recursos: gestión de recursos humanos (4), gestión económico-financiera (5), gestión de la información (6) y gestión de recursos materiales (7). Así como tres denominamos procesos avanzados de gestión: gestión de las relaciones (8), gestión de la estructuración (9) y gestión del aprendizaje (10).

Esta implementación se basa en el modelo de calidad European Foundation for Quality Management (EFQM) que permite orientar las acciones hacia los resultados, el cliente y el liderazgo, direccionando la gestión hacia procesos de aprendizaje continuo, innovación y mejoras, a través de alianza y desarrollo de responsabilidad social de la organización (figura 2).

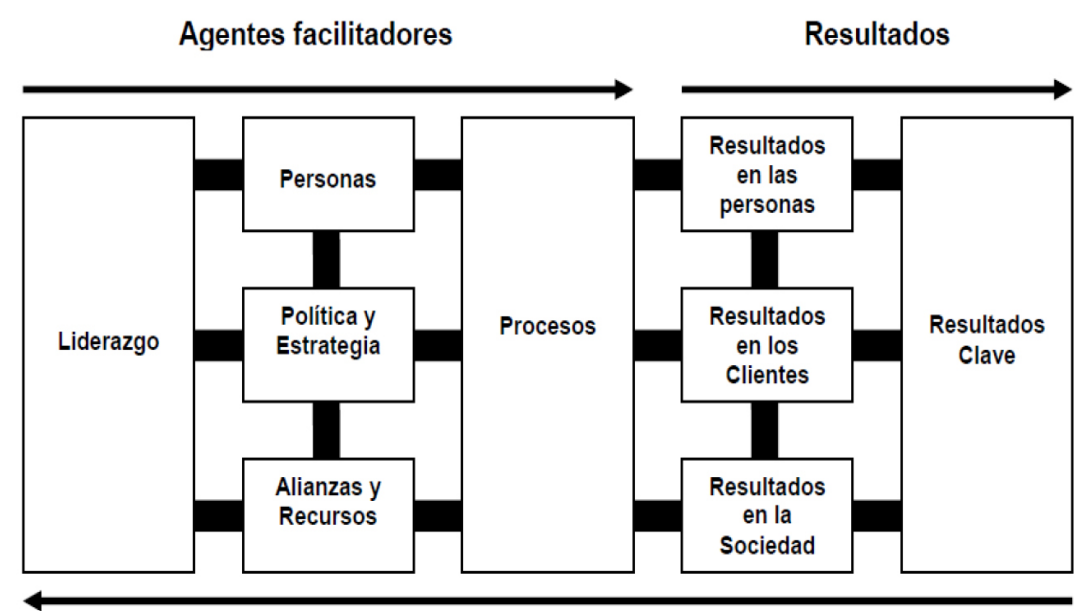

Innovación y Aprendizaje

Figura 2. Modelo de Calidad EFQM.

Fuente: (Fantova, 2005)
El modelo direcciona la manera como aprovechar la creatividad y la innovación, desarrollando la capacidad organizacional, para alcanzar el éxito del talento humano obteniendo resultados que se mantengan en el tiempo que permitan a la organización liderar proyectos sostenibles. (Fantova, 2005)

\section{Modelo de planeación y control integral de la gestión municipal (PODER -SEEO)}

(Martínez Fajardo, 2004) realiza una propuesta del modelo basado en un caso excepcional de gestión exitosa en Colombia. La experiencia de gestión estaba orientada con los criterios de participación, motivación y auto organización de la comunidad.

Este modelo se diseñó alrededor de tres componentes que permitían realizar las funciones de planeación y control en función de la eficacia social. Los componentes establecidos para alcanzar la misma en concordancia con la aplicación del modelo están contenidos en la producción de servicios públicos, manejo de recursos humanos, financieros, físicos y la participación de la comunidad e interacción con red de organizaciones; las siglas de este modelo la componen de la siguiente forma: Planeación, Organización, Direccionamiento, Evaluación, Retroalimentación se combinan con un Sistema de Evaluación de Eficacia Organizacional.(González \& Rodríguez, 2010)

La metodología aplicada para los Indicadores de Planeación y Evaluación de eficacia social son: 1. Capacidad de gestión y productividad, 2. Capacidad del funcionario público, 3. Capacidad y efectividad del presupuesto, 4. Capacidad de direccionamiento de la investigación tecnológica, y 5 . Efectividad de interacción con variables 
externas, los que permiten lograr una evaluación de la eficacia de la gestión realizada.(Kaplan \& Norton, 2009)

\section{METODOLOGÍA}

La exigencia actual de la educación superior, no solo a nivel país, sino en Latinoamérica y el mundo, conlleva a obtener resultados sólidos y sostenibles respecto al bien más preciado, el ser humano, lo que significa que un estudiante debe forjarse no solo bajo el campo del conocimiento, sino también de la producción de propuestas que permitan mejorar a la sociedad en todos sus ámbitos. Es por esto, que hablar de la educación superior, actualmente es referirse a la gestión que se realiza bajo la unificación de los ejes estructurales como son (figura 3):

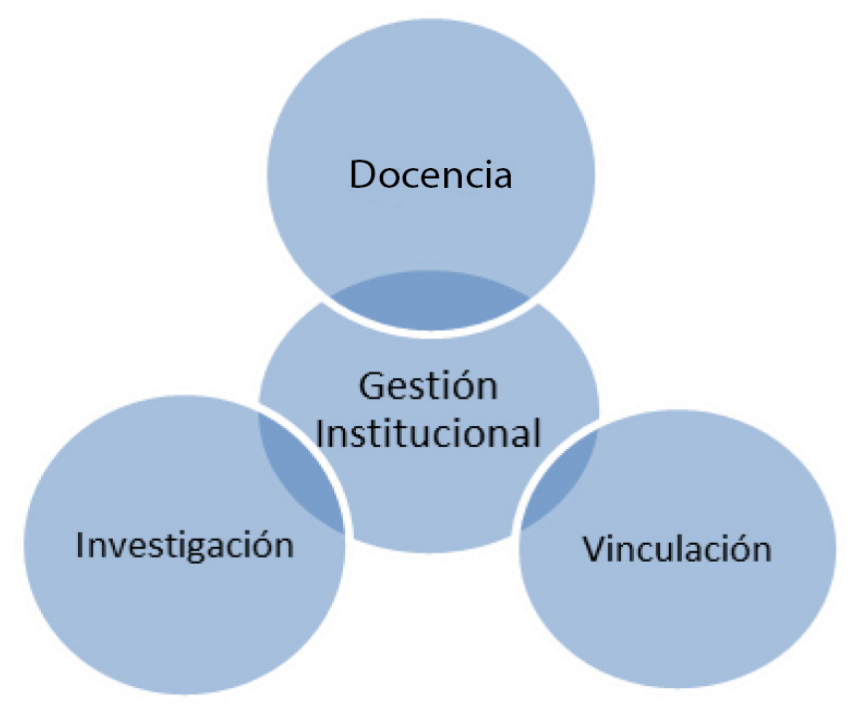

Figura 3. Ejes estructurales de la Educación Superior.

Fuente: Ley Orgánica de Educación Superior y Reglamento de Régimen Académico (LOES, 2010)
En concordancia con los elementos mostrados en los modelos de gestión social referidos en el marco teórico, se tomaron las características fundamentales de cada uno de ellos; así como, se apuntó hacia las exigencias de la calidad de la Educación Superior y las necesidades institucionales y sociales, ante ello, el Modelo de Gestión DVS-ULVR que se propone, se basa en la integralidad de los actores, en elementos que se complementan entre sí y que son aplicables a nivel de las Instituciones de Educación Superior (IES).

Esta propuesta se construye sobre ocho pilares fundamentales:

1. Unificación de criterios: para la concepción de los proyectos de vinculación: Interdisciplinarios, Multidisciplinarios e Interinstitucionales.

2. Planeación del Proyecto:

a. creación de línea base

b. identificación de la zona de influencia

c. determinación de problemas reales;

d. aplicación de la matriz de marco lógico;

e. metodología de investigación; y,

f. estudio de viabilidad y sostenibilidad.

3. Aplicación de formatos: de acuerdo a los indicadores de evaluación, se fundamentan en siete componentes (figura 4): 
-Datos Generales

- Apertura a la Equidad e

Igualdad

- Cobertura y Localización

- nstituciones Involucradas

- Personal Responsable

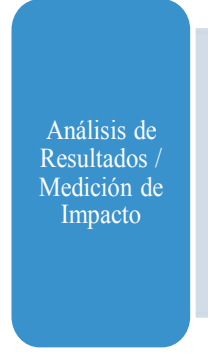

- Matriz de

Indicadores por

- Proyección de

Impacto

- Indicadores

Especificos acorde

a naruraleza de

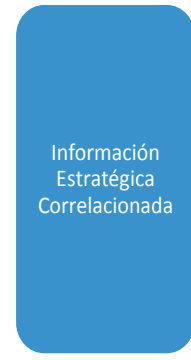

- Objetivos Plan Nacional Buen Vivir

- Objetivos Plan Nacional de Desarrolla

- Objetivos Plan Estratégico

Desarrollo Institucional

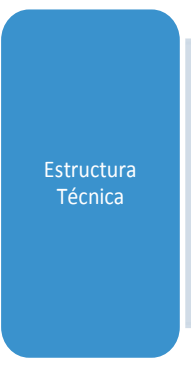

- Línea Base

- Árbol de Problemas

- Árbol de Objetivos

- Cuadro de Involucrados

- Matriz Marco Lógico

- Metodología de Investigación

- Viabilidad y Sostenibilidad

- Diagramas de Ejecución
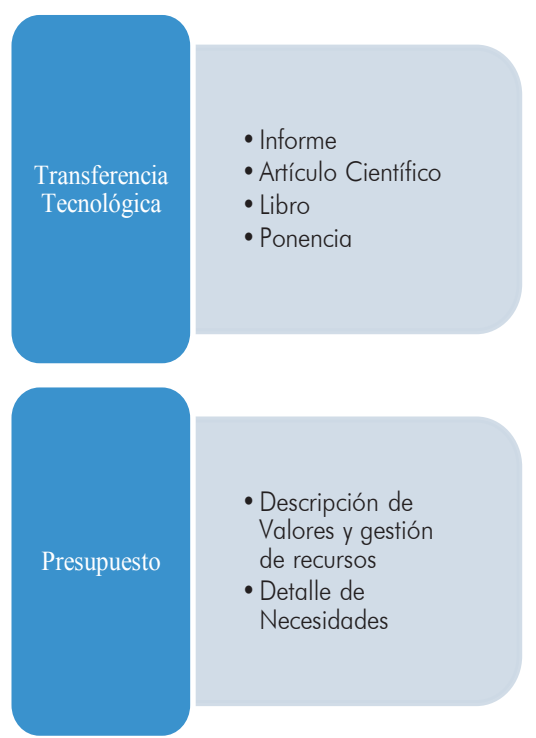

Estudiantes

- Nombres y Apellidos

Vinculados

- Número de Horas

- Calificación

Figura 4. Componentes de indicadores de evaluación. Fuente: Normativa Departamento de Vinculación ULVR.

4. Medición de Resultados: basados en 5 dimensiones relacionadas a los objetivos institucionales macros de vinculación (Tablas 1, 2, 3, 4 y 5):
Tabla 1

Tabla de Indicadores Dimensión Económica Empresarial.

\begin{tabular}{|c|c|}
\hline \multicolumn{2}{|c|}{ DIMENSIÓN ECONÓMICA EMPRESARIAL } \\
\hline \multicolumn{2}{|r|}{ Indicador } \\
\hline Nombre & Fórmula \\
\hline $\begin{array}{l}\text { Tipos de Actividades } \\
\text { Económicas }\end{array}$ & $\begin{array}{l}\text { Cantidad de actividades económicas identificadas y } \\
\text { segregadas por tipos de parámetros de medición / Total } \\
\text { actividades económicas identificadas }\end{array}$ \\
\hline $\begin{array}{c}\text { Participación e } \\
\text { Integración de Actores } \\
\text { y Recursos }\end{array}$ & $\begin{array}{l}\text { \# de actores económicos integrados al proyecto / } \\
\text { total de actores económicos de la zona de intervención }\end{array}$ \\
\hline \multirow{5}{*}{$\begin{array}{c}\text { Situación Productiva de } \\
\text { los Actores }\end{array}$} & Volumen de Ventas Generadas / Volumen de producción \\
\hline & $\begin{array}{c}\text { Volumen de Ventas Generadas / Volumen de ventas } \\
\text { Programadas }\end{array}$ \\
\hline & $\begin{array}{c}\text { Volumen Total de Ventas / Cantidad de Recursos } \\
\text { Humanos }\end{array}$ \\
\hline & $\begin{array}{c}\text { Monto de Inversión en mejoramiento y adquisición de } \\
\text { equipos y maquinarias / Costo histórico contable de } \\
\text { equipos y maquinarias }\end{array}$ \\
\hline & $\begin{array}{c}\text { Monto de Inversión en mejoramiento y adquisición de } \\
\text { sistemas informáticos / Costo histórico contable de } \\
\text { sistemas informáticos }\end{array}$ \\
\hline Capacitación & $\begin{array}{c}\text { Número de actores capacitados / Total de actores } \\
\text { de la zona de intervención }\end{array}$ \\
\hline Asesoría & $\begin{array}{l}\text { \# de actividades económicas asesoradas / Total de } \\
\text { Actividades económicas identificadas }\end{array}$ \\
\hline Desarrollo de Proyectos & Cantidad de Proyectos desarrollados por la intervención \\
\hline \multirow{5}{*}{$\begin{array}{l}\text { Mejoramiento Económico } \\
\text { de los Actores }\end{array}$} & Ingresos Actuales / Ingresos Anteriores \\
\hline & Costos actuales / Costos anteriores \\
\hline & Gastos Actuales / Gastos Anteriores \\
\hline & Resultados Actuales / Resultados Anteriores \\
\hline & $\begin{array}{c}\text { Declaraciones Tributarias erradas / Declaraciones } \\
\text { Tributarias Totales }\end{array}$ \\
\hline
\end{tabular}

Fuente: Matriz de Análisis de Resultados y Medición de Impacto ULVR. 
Tabla 2

Tabla de Indicadores Dimensión Social.

\begin{tabular}{|c|c|}
\hline \multicolumn{2}{|r|}{ DIMENSIÓN SOCIAL } \\
\hline \multicolumn{2}{|r|}{ Indicador } \\
\hline Nombre & Fórmula \\
\hline $\begin{array}{l}\text { Identificación } \\
\text { de Necesidades }\end{array}$ & $\begin{array}{c}\text { Datos comparativos de hasta los últimos } \\
3 \text { años de necesidades en la } \\
\text { situación específica }\end{array}$ \\
\hline \multirow{6}{*}{$\begin{array}{l}\text { Plan de Intervención para } \\
\text { el mejoramiento de la } \\
\text { calidad de vida }\end{array}$} & $\begin{array}{l}\text { \# de Objetivos cumplidos / } \\
\text { \# de Objetivos Totales }\end{array}$ \\
\hline & \# de Metas cumplidas / \# de Metas Totales \\
\hline & $\begin{array}{l}\text { Monto de Recursos Utilizados / } \\
\text { Monto de Presupuesto del Proyecto }\end{array}$ \\
\hline & $\begin{array}{c}\text { \# de Actores Vinculados de las zonas } \\
\text { de intervención / \# de actores totales de } \\
\text { las zonas intervenidas }\end{array}$ \\
\hline & $\begin{array}{l}\text { \# de Situaciones Ejecutadas / } \\
\text { Total de Situaciones Diagnosticadas }\end{array}$ \\
\hline & $\begin{array}{l}\text { \# de Acciones Cumplidas / } \\
\text { \# de Acciones Planificadas }\end{array}$ \\
\hline $\begin{array}{l}\text { Identificación de } \\
\text { Organizaciones de apoyo }\end{array}$ & $\begin{array}{c}\text { Cantidad de organizaciones de apoyo } \\
\text { detectadas a las cuales puedan incorporarse } \\
\text { las zonas de intervención }\end{array}$ \\
\hline $\begin{array}{c}\text { Socialización de Información } \\
\text { de Organizaciones de apoyo } \\
\text { detectadas }\end{array}$ & $\begin{array}{l}\text { Información socializada / } \\
\text { información obtenida }\end{array}$ \\
\hline \multirow{4}{*}{$\begin{array}{c}\text { Construcción de necesidades } \\
\text { de participación/creación de } \\
\text { Organizaciones de apoyo }\end{array}$} & $\begin{array}{l}\text { \# de necesidades de participación } \\
\text { necesidades de participación identificadas }\end{array}$ \\
\hline & Participaciones en organizaciones de apoyo \\
\hline & $\begin{array}{c}\text { \# de participantes en organizaciones de } \\
\text { apoyo / \# de actores vinculados de } \\
\text { la zona de influencia }\end{array}$ \\
\hline & Cantidad de organizaciones de apoyo creadas \\
\hline
\end{tabular}

\begin{tabular}{|c|c|}
\hline Información secundaria & $\begin{array}{c}\text { Total de datos obtenidos de } \\
\text { información secundaria }\end{array}$ \\
\hline Información Faltante & Total de datos faltantes obtenidos \\
\hline Línea Base & $\begin{array}{c}\text { Datos Integrados en la construcción } \\
\text { de la línea base/Total de datos recopilados }\end{array}$ \\
\hline
\end{tabular}

Fuente: Matriz de Análisis de Resultados y Medición de Impacto ULVR.

Tabla 3

Tabla de Indicadores Dimensión Infraestructura Territorial y Urbanismo.

\begin{tabular}{|c|c|}
\hline \multicolumn{2}{|c|}{ DIMENSIÓN INFRAESTRUCTURA TERRITORIAL Y URBANISMO } \\
\hline \multicolumn{2}{|c|}{ Indicador } \\
\hline Nombre & Fórmula \\
\hline $\begin{array}{c}\text { Infraestructura de Servicios } \\
\text { básicos }\end{array}$ & $\begin{array}{c}\text { Datos comparativos de hasta los últimos } 3 \text { años de } \\
\text { infraestructura de los servicios básicos acorde a la } \\
\text { naturaleza de cada proyecto. }\end{array}$ \\
\hline $\begin{array}{l}\text { Infraestructura de espacios } \\
\text { urbanos }\end{array}$ & $\begin{array}{l}\text { Datos comparativos de hasta los últimos } 3 \text { años de } \\
\text { infraestructura de los espacios urbanos acorde a la } \\
\text { naturaleza de cada proyecto. }\end{array}$ \\
\hline \multirow{6}{*}{$\begin{array}{l}\text { Plan de intervención de } \\
\text { infraestructura de servicios } \\
\text { básicos y espacios urbanos }\end{array}$} & \# de Objetivos cumplidos / \# de Objetivos Totales \\
\hline & \# de Metas cumplidas / \# de Metas Totales \\
\hline & $\begin{array}{l}\text { Monto de Recursos Utilizados / } \\
\text { Monto de Presupuesto del Proyecto }\end{array}$ \\
\hline & $\begin{array}{c}\text { \# de Actores Vinculados de las zonas } \\
\text { de intervención / \# de actores totales } \\
\text { de las zonas intervenidas }\end{array}$ \\
\hline & $\begin{array}{l}\text { \# de Situaciones ejecutadas / } \\
\text { Total de Situaciones Diagnosticadas }\end{array}$ \\
\hline & \# de Acciones Cumplidas / \# de Acciones Planificadas \\
\hline $\begin{array}{c}\text { Necesidades de infraestructuro } \\
\text { y urbanismo }\end{array}$ & $\begin{array}{c}\text { Cantidad de necesidades de infraestructura } \\
\text { y urbanismo identificadas }\end{array}$ \\
\hline Participación comunitaria & $\begin{array}{l}\text { \# de actores participantes / } \\
\text { total de actores de la zona de influencia }\end{array}$ \\
\hline
\end{tabular}

Fuente: Matriz de Análisis de Resultados y Medición de Impacto ULVR. 
Tabla 4

Tabla de Indicadores Dimensión Educativa.

\begin{tabular}{|c|c|}
\hline \multicolumn{2}{|c|}{ DIMENSIÓN EDUCATIVA } \\
\hline \multicolumn{2}{|r|}{ Indicador } \\
\hline Nombre & Fórmula \\
\hline \multirow{3}{*}{$\begin{array}{c}\text { Planificación de los procesos } \\
\text { educativos. }\end{array}$} & Cantidad de necesidades educativas detectadas. \\
\hline & \# de acciones proyectadas / necesidades detectadas \\
\hline & Orden lógico en que se presentan las acciones. \\
\hline $\begin{array}{l}\text { Ejecución de los procesos } \\
\text { educativos }\end{array}$ & \# de acciones ejecutadas / \# de acciones planificadas. \\
\hline \multirow{2}{*}{$\begin{array}{c}\text { Evaluación de los procesos } \\
\text { educativos }\end{array}$} & Información rendida periódicamente. \\
\hline & Información rendida / información solicitada \\
\hline \multirow{3}{*}{$\begin{array}{l}\text { Difusión de conocimientos, } \\
\text { experiencia y habilidades } \\
\text { con el fin de facilitar su uso, } \\
\text { aplicación y explotación }\end{array}$} & Conocimiento empleado / conocimiento generado \\
\hline & Problemáticas solucionadas / conocimiento generado \\
\hline & $\begin{array}{l}\text { Cantidad de acciones producidas } \\
\text { / conocimiento generado }\end{array}$ \\
\hline
\end{tabular}

Fuente: Matriz de Análisis de Resultados y Medición de Impacto ULVR.

\section{Tabla 5}

Tabla de Indicadores Dimensión Ambiental.

\begin{tabular}{|c|c|}
\hline \multicolumn{2}{|c|}{ DIMENSIÓN AMBIENTAL } \\
\hline Nombre & Indicador \\
\hline $\begin{array}{c}\text { Valor de impacto ambiental del } \\
\text { proyecto. }\end{array}$ & Resultado de la evaluacióndel impacto ambiental. \\
\hline $\begin{array}{c}\text { Valoración ambiental de la } \\
\text { zona de estudio. }\end{array}$ & Resultado de la evaluación del impacto ambiental. \\
\hline
\end{tabular}

Fuente: Matriz de Análisis de Resultados y Medición de Impacto ULVR.
1. Relación de Proyectos con Líneas de Investigación Vinculación Institucional.

2. Dirección de la Gestión: hacia los actores activos.

3. Implantación del Sistema de Control: desde la concepción de los proyectos, durante el desarrollo y la culminación de estos.

4. Retroalimentación: de los resultados obtenidos tanto con los actores principales institucionales, como de la zona de influencia.

El modelo propuesto es la base esencial sobre el cual se ha desarrollado la gestión académica, investigativa y administrativa de la Vinculación con la Sociedad, por lo que, la importancia de su aplicación, se fundamenta en poseer una estructura metodológica y organizativa, permitiendo la cohesión entre la sociedad, los organismos públicos y privados; y, las instituciones de educación superior, respecto a los logros que se alcancen tanto de manera inmediata a través de sus resultados, así como, el impacto que se genere en el tiempo.

\section{RESULTADOS}

La aplicación del Modelo durante el año 2018 se lo realizó en la Universidad Laica Vicente Rocafuerte de Guayaquil, con la finalidad de conocer la eficiencia de su construcción; y, con los resultados obtenidos, determinar la viabilidad de su aplicación en momentos distintos a los que fueron desarrollados (Tabla 6). 
Modelo de Gestión para proyectos interdisciplinarios de Vinculación

José E. Pazmiño E., Luis E. Cortez A., Linda C. Maldonado G. • VínCULOS-ESPE (2019) VOL. 4, No.3:9-19

Tabla 6.

Pilares Fundamentales Modelo DVS-ULVR.

\begin{tabular}{|c|c|c|}
\hline Pilares Fundamentales & $\begin{array}{l}\text { Herramientas } \\
\text { Metodológicas }\end{array}$ & Resultados Obtenidos \\
\hline 1. Unificación de criterios & $\begin{array}{l}\text { Mesas de Trabajo con Actores } \\
\text { Institucionales }\end{array}$ & $\begin{array}{l}\text { Actas de Trabaio: } \\
\text { - Análisis de Proyectos } \\
\text { Disciplinarios, } \\
\text { Interdisciplinarios y } \\
\text { Multidisciplinarios } \\
\text { - Sistematización } \\
\text { Propuestas } \\
\text { - Unificación de criterios para } \\
\text { la planeación }\end{array}$ \\
\hline 2. Planeación del Proyecto & $\begin{array}{l}\text { Análisis de la Zona de Influencia } \\
\text { Árbol de Problemas }\end{array}$ & $\begin{array}{l}\text { Planificación de proyectos de } \\
\text { vinculación en las zonas de } \\
\text { influencia } \\
\text { Gestión de Convenios } \\
\text { Matriz de Marco Lógico }\end{array}$ \\
\hline 3. Aplicación de formatos & $\begin{array}{l}\text { Guía de Procedimientos para la } \\
\text { Formulación, Presentación y } \\
\text { Aprobación de Proyectos de } \\
\text { Vinculación con la Sociedad- } \\
\text { ULVR } \\
\text { Tabla de Valoración para } \\
\text { Aprobación de Proyectos }\end{array}$ & Informe de Aprobación \\
\hline & $\begin{array}{l}\text { Guías de } \\
\text { Informes Inicial, Mensución de y Final }\end{array}$ & $\begin{array}{l}\text { Informes iniciales, mensuales y } \\
\text { finales elaborados por cada } \\
\text { proyecto de vinculación }\end{array}$ \\
\hline 4. Medición de Resultados & $\begin{array}{l}\text { Matriz de Análisis de Resultados } \\
\text { y Medición de Impacto }\end{array}$ & $\begin{array}{l}\text { Indicadores Institucionales y de } \\
\text { Indicadores Específicos }\end{array}$ \\
\hline $\begin{array}{l}\text { 5. Relación de Proyectos con } \\
\text { Líneas de Investigación - } \\
\text { Vinculación Institucionales }\end{array}$ & $\begin{array}{llr}\text { Estatutos y reglamentaciones de } \\
\text { educación } & \text { superior } & \text { e } \\
\text { institucionales } & \end{array}$ & $\begin{array}{l}\text { Aplicación de las líneas de } \\
\text { investigación y vinculación: } \\
\text { Línea 1. Formación integral, } \\
\text { atención a la diversidad y } \\
\text { educación inclusiva. }\end{array}$ \\
\hline
\end{tabular}

\begin{tabular}{|c|c|c|}
\hline & & $\begin{array}{l}\text { Línea 2. Sociedad civil, derechos } \\
\text { humanos y gestión de la } \\
\text { comunicación. } \\
\text { Línea 3. Territorio, medio } \\
\text { ambiente y materiales } \\
\text { innovadores para la construcción. } \\
\text { Línea 4. Desarrollo estratégico } \\
\text { empresarial y emprendimientos } \\
\text { sustentables. }\end{array}$ \\
\hline 6. Dirección de la Gestión & $\begin{array}{l}\text { Plan Operativo Anual } \\
\text { Instructivo de Proyectos de } \\
\text { Vinculación }\end{array}$ & $\begin{array}{l}\text { Evidencias de los Procesos de } \\
\text { gestión anuales del Departamento } \\
\text { de Vinculación } \\
\text { Directrices emitidas por el } \\
\text { Departamento de Vinculación, } \\
\text { para la toma de decisiones }\end{array}$ \\
\hline $\begin{array}{l}\text { Implantación del Sistema de } \\
\text { Control }\end{array}$ & $\begin{array}{l}\text { Mesas de Trabajo con actores } \\
\text { principales } \\
\text { Revisión de Informes inicial, } \\
\text { mensual y final }\end{array}$ & $\begin{array}{l}\text { Fichas y Tablas de Medición de } \\
\text { avances de cumplimiento de } \\
\text { objetivos, metas y resultados de } \\
\text { los proyectos } \\
\text { Evidencias de las evaluaciones } \\
\text { mensuales }\end{array}$ \\
\hline Retroalimentación & Informes de Resultados & $\begin{array}{l}\text { Socialización de los resultados } \\
\text { con los actores internos y de la } \\
\text { zona de influencia } \\
\text { Direccionamiento In al } \\
\text { Departamento de Investigación } \\
\text { para la continuación de los } \\
\text { proyectos }\end{array}$ \\
\hline
\end{tabular}

\section{CONCLUSIONES}

1. Los proyectos de vinculación se ejecutaban de manera disciplinaria, sin existir una articulación homogénea de objetivos y metodología de procesos; así como, no existían direccionamientos específicos por parte de los organismos de control de educación superior sobre esta temática. 
2. La necesidad institucional de llevar a cabo proyectos de vinculación interdisciplinarios motivó la construcción del Modelo de Gestión DVS-ULVR.

3. El Modelo de Gestión DVS-ULVR apunta a la optimización del uso del talento humano, la distribución de recursos financieros, y el análisis de resultados para la medición de impactos, en cumplimiento de la responsabilidad social e institucional.

4. La aplicación de este modelo permitió la motivación y el compromiso de los actores institucionales hacia los actores de la sociedad y viceversa, producto de la interdisciplinariedad, la eficiencia de los procesos; y, la unificación de criterios de desarrollo de los proyectos de vinculación efectuados por la Universidad en el 2018.

\section{RECOMENDACIONES}

1. Adaptar el Modelo de Gestión DVS-ULVR, como guía metodológica para la ejecución de proyectos de vinculación.

2. Socializar con las IES el modelo de gestión DVS-ULVR y sus resultados, para obtener información necesaria que permita, mediante la retroalimentación, la mejora del mismo.

3. Realizar un seguimiento a los proyectos de vinculación desarrollados por la ULVR, a fin de conocer el impacto en el tiempo de estos y la capacidad de réplica que han tenido en la solución de problemas sociales, lo que validará la implantación del modelo.

4. Aplicar el modelo desarrollado en la solución de otros problemas sociales, a fin de garantizar su efectividad y eficiencia en ambientes distintos al académico.

\section{BIBLIOGRAFÍA}

Fantova, F. (2005). Nuevos modelos en gestión social: calidad y excelencia en las organizaciones sociales. Congreso Internacional de Calidad de Vida En Personas Con Discapacidad, 25. Retrieved from fantova.net/?wpfb_dl=97

González, J., \& Rodríguez, M. (2010). Modelos de Capital Intelectual y sus indicadores en la universidad pública, (11).

Kaplan, R. S., \& Norton, D. P. (2009). La vinculación de los indicadores del cuadro de mando integral. El Cuadro de Mando Integral: The Balanced Scorecard, 187-208. Retrieved from https://core.ac.uk/ download/pdf/90646972.pdf

LOES. Ley Orgánica de Educación Superior (2010). Ecuador.

Martínez Fajardo, C. E. (2004). Management and creating knowledge. Innovar, 14(23), 13-23. Retrieved from http:// www.scielo.org.co/scielo.php? script=sci_arttext\&pid=S0121 $50512004000100001 \& \operatorname{lng}=e n \& n r m=i s o \& t \operatorname{lng}=e s$

Royero, J. (2009). Modelo Integrado de Control de Gestión (MICG). (El Cid Editor, Ed.) (6a ed.). Santa Fe, Argentina. 